\title{
28 Research Square \\ Gender as a factor affecting the NK cell activity in patients successfully treated for chronic hepatitis C with DAA
}

Agata Zientarska ( $\sim$ agatakierepa@gmail.com )

Poznan University of Medical Sciences

\section{Aleksandra Witkowska}

Poznan University of Medical Sciences

Błażej Rozpłochowski

Poznan University of Medical Sciences

Justyna Mikuła-Pietrasik

Poznan University of Medical Sciences

Mariusz Kaczmarek

Poznan University of Medical Sciences

Arleta Kowala-Piaskowska

Poznan University of Medical Sciences

Krzysztof Książek

Poznan University of Medical Sciences

Jan Żeromski

Poznan University of Medical Sciences

Iwona Mozer-Lisewska

Poznan University of Medical Sciences

\section{Research Article}

Keywords: Chronic hepatitis C (CHC), NK cell, directly acting antivirals (DAA)

Posted Date: November 20th, 2020

DOl: https://doi.org/10.21203/rs.3.rs-110558/v1

License: (c) (i) This work is licensed under a Creative Commons Attribution 4.0 International License.

Read Full License 


\section{Abstract}

Chronic hepatitis $\mathrm{C}(\mathrm{CHC})$ affects the activity of NK cells, but successful interferon-free treatment partially restores it. The goal of the study was to assess whether gender influences those alterations. We examined 21 women after menopause and 24 men with $\mathrm{CHC}$ treated with directly acting antivirals (DAA), and 33 healthy volunteers. With flow cytometry, we analysed KIR2DS4, NKG2D, NKp30, KIR2DL2/DL3, NKG2A, TRAIL and granzyme B on the surface of NK cells, simultaneously we checked serum CXCL10 with ELISA. Overall, patients with CHC had higher expression of KIR2DS4, NKG2A, NKp30 and a lower percentage of NK cells among lymphocytes than the control group. After the treatment KIR2DS4, KIR2DL2/DL3 and NKG2A, TRAIL NKp30 on NK cells decreased, while the percentage of NK cells, expression of granzyme B and NKG2D increased. Serum CXCL10 was elevated before the treatment and dropped afterwards. We observed differences between genders in the expression of KIR2DL2/DL3 (higher in female) and NKp30 (elevated in men) comparing $\mathrm{CHC} /$ control groups. After the treatment of KIR2DL2/DL3, NKp30 and CXCL10 dropped only in female while granzyme B increased in male. In conclusion, the response of NK cells among men and women in post-menopausal age with $\mathrm{CHC}$ differs. Our research may lead to more studies on different nature of female and male immune systems in the context of HCV infection and treatment.

\section{Introduction}

Natural killer cells are considered a crucial element of the innate immune response against viral infections. We divide their effector functions into two main categories. First, they present direct cytotoxicity either by releasing cytotoxic granules containing perforin and granzymes or inducing death receptor-mediated apoptosis by expressing Fas ligand or TNF-related apoptosis-inducing ligand (TRAIL) ${ }^{1}$. Second, NK cells produce pro-inflammatory and immunosuppressive cytokines as well as chemokines ${ }^{2}$.

To activate their effector functions, NK cells do not possess antigen-specific receptors nor require antigen presentation. The process is regulated by several inhibitory or activating surface receptors and their ligands on target cells. On healthy cells, MHC class I molecules prevent mobilisation and cytotoxicity of NK cells via inhibitory KIR receptors. Virus-infected cells usually decrease MHC class I expression and increase the presentation of stimulating ligands ${ }^{3}$.

Chronic hepatitis $\mathrm{C}(\mathrm{CHC})$ does not simply lead to increased activation of NK cells but produces a dysregulation of their functions instead ${ }^{4}$. Expression of particular activating and inhibiting receptors on NK cells is distorted; nevertheless, the involved authors show various results of their study, as presented in table 1. 


\begin{tabular}{|l|l|l|l|}
\hline & NK effector function & Chronic & After DAA treatment \\
\hline $\begin{array}{l}\text { KIR2DS4 } \\
\text { (CD158i) }\end{array}$ & Activating receptor & $\leftrightarrow^{56}$ & $?$ \\
\hline NKG2D & Activating receptor & $\begin{array}{l}\uparrow^{7} \\
\leftrightarrow^{8} \\
\downarrow^{91011}\end{array}$ & $\leftrightarrow^{128}$ \\
\hline NKp30 & Activating receptor & $\begin{array}{l}\uparrow^{121114} \\
\downarrow^{1516}\end{array}$ & $\downarrow^{12} 8_{14}$ \\
\hline $\begin{array}{l}\text { KIR2DL2/DL3 } \\
\text { (CD158b) }\end{array}$ & Inhibiting receptor & $\begin{array}{l}\uparrow^{9} 1614 \\
\leftrightarrow\end{array}$ & $\downarrow^{14}$ \\
\hline NKG2A & Inhibiting receptor & $\uparrow^{58151714}$ & $\downarrow^{814}$ \\
\hline TRAIL & Direct cytotoxicity & $\uparrow^{1217}$ & $\downarrow^{12} 8$ \\
\hline Granzyme B & Cytotoxic granules & $\uparrow^{18}$ & $\begin{array}{l}\uparrow^{18}-\text { combined therapy (with IFN) } \\
\downarrow^{18}\end{array}$ \\
\hline CXCL10 & Chemokine & $\uparrow^{8}$ & $\downarrow^{8}$ \\
\hline
\end{tabular}

Table 1 Porameters of NK cells examined in the current study, assessed by other authors. Arrows indicate alterotions observed among chronically HCV-infected patients and changes after the successful therapy with direct-acting antivirals (DAA).

Female and male immune systems differ in many aspects. Causes of sexual dimorphism are both hormonal and genetic. Females develop enhanced innate and adaptive immune responses comparing to males, which makes them less predisposed to various infections and malignancies, but more susceptible to autoimmune diseases ${ }^{20}$. Regarding NK cells, males have a higher count of NK cells, NK56dim cells proportion and higher expression of $\mathrm{CD}^{2} 7^{21}$. In women of reproductive age estrogens inhibit NK cells cytotoxicity at the ovulatory phase ${ }^{22}$, but in older age women have more vigorous cytotoxic activity than men $^{23}$. Among chronically HBV-infected patients, intrahepatic NK cells have higher degranulation activity in women and correlate with estradiol level ${ }^{24}$.

Gender disparity also has an impact on prognosis in $\mathrm{CHC}$. Immunological differences combine with hepatoprotective effects of estradiol and estrogen ${ }^{25}$. As a result, in HCV-associated cirrhosis male patients with hepatocellular carcinoma (HCC) have a worse outcome than women ${ }^{26}$. Short HCV infections treated with interferon-based therapies had higher chances of success in female ${ }^{27}$. Among $\mathrm{HCV}$-infected patients treated with direct-acting antivirals, $\mathrm{HCC}$ recurrence and occurrence rate is higher among men ${ }^{28}$.

The goal of the current study was to investigate whether gender influences changes observed in NK cells of chronically HCV-infected patients cured with the novel, interferon-free therapies.

\section{Materials And Methods}

We collected a study group from among patients treated at the Department of Infectious Diseases, Hepatology and Acquired Immunodeficiencies of Poznan University of Medical Sciences. It consisted of 45 patients with $\mathrm{CHC}$ genotype $1 \mathrm{~b}$ qualified to DAA treatment, 21 female and 24 male with different stages of liver fibrosis. To eliminate the impact of rapid hormonal changes in the course of the menstrual cycle, we decided to qualify only female after menopause. Exclusion criteria were: the presence of HCC, 
HIV co-infection, presence of HBsAg, administering of any immunomodulatory or immunosuppressive drugs.

We tested the subjects up to 7 days before the treatment (TO) and twice after the completed regimen - an average of 200 days after the first dose (T1) and 167 days later (T2). Twenty-two of patients were treated with sofosbuvir/ledipasvir (among them 16 regiments with ribavirin), 7 received ombitasvir/paritaprevir/ritonavir/dasabuvir, four grazoprevir/elbasvir, eight sofosbuvir/velpatasvir and four got glecaprevir/pibrentasvir. We applied the regiments according to the polish recommendations ${ }^{29}$. All the patients completed the therapy and achieved a sustained virological response (SVR). Then, we enrolled a control group that consisted of 33 volunteers without HCV infection, 22 men and 11 women in post-menopausal age.

We measured liver stiffness with transient elastography technique, using FibroScan Compact device (Echosens, Paris, France) in compliance with the manufacturer's instructions ${ }^{30}$.

Cell immunophenotyping was performed by flow cytometry with the use of direct fluorescence method, which we described in our previous study ${ }^{31}$. Briefly, the appropriate monoclonal antibodies fluorochromelabelled (described in Table 2) were added to the cytometric tubes at a rate of $5 \mathrm{ml}$ each. $100 \mathrm{ml}$ of peripheral blood mixed with EDTA were added to the test tubes. Samples were mixed gently on vortex and incubated for $15 \mathrm{~min}$ at room temperature protected from light. After this time, $500 \mathrm{ml}$ of erythrocyte lysis buffer (Becton Dickinson) were added to the test tubes and incubated for $10 \mathrm{~min}$. Lysis was stopped by adding PBS solution (phosphate-buffered saline) and centrifuged. This step was repeated. Granzyme B was studied intracellular after additional permeabilisation of cells with Perm/Wash buffer (BD Biosciences). All obtained cell pellets were resuspended in PBS buffer and were acquired using a FACS Canto flow cytometer (Becton Dickinson). The obtained results were analysed with the FACS Diva software (Becton Dickinson), integrated with the cytometer. For each examined antibody percent of positive cells and Mean Fluorescence Intensity (MFI) was determined.

Table 2. Antibodies used in the study for cell immunophenotyping.

\begin{tabular}{l|l|l|l}
\multicolumn{2}{l}{ Antibody } & \multicolumn{1}{l}{ Fluorochrome } & \multicolumn{1}{l}{ Clone } \\
\hline CD158b (KIR2DL2/DL3) & PE & DX27 & Miltenyi Biotec \\
\hline CD158i (KIR2DS4) & PE & JJC11.6 & Miltenyi Biotec \\
\hline CD159a (NKG2A) & PE & REA110 & Miltenyi Biotec \\
\hline CD314 (NKG2D) & PE & BAT221 & Miltenyi Biotec \\
\hline CD337 (NKp30) & PE & AF29-4D12 & Mitenyi Biotec \\
\hline CD253 (TRAIL) & PE & RIK-2 & BD Pharmingen $^{\text {TM }}$ \\
\hline Granzyme B & PE & GB11 & BD Pharmingen $^{\text {TM }}$ \\
\hline
\end{tabular}

Concentration of human CXCL10/IP-10 in serum was measured using appropriate DuoSet $\circledast$ Immunoassay Development kits (R\&D Systems, Minneapolis, USA), according to manufacturer's instructions. 
The statistical analysis was conducted using STATISTICA software version 13.0 (StatSoft, Cracow, Poland). Since in Shapiro-Wilk test we evaluated most of our variables to not be normally distributed, we were using non-parametrical tests. We analysed dependent variables with Wilcoxon signed-rank test and non-dependent ones with Mann-Whitney U test. A p-value $\leq 0.05$ was considered statistically significant 32 .

\section{Results}

Among the patients qualified to the study, $66,7 \%$ had radiological and biochemical indicators of liver cirrhosis, confirmed in FibroScan. There were no significant differences between genders in assessed parameters of the liver function nor basic biochemical and morphological parameters, presented in table 3. After the completed treatment hepatic stiffness, alanine transaminase (ALT) aspartate transaminase (AST) and total bilirubin level decreased significantly ( $p=0,002,0,00002,0,00002$ and 0,007 , respectively) while platelet count (PLT) and white blood cells count (WBC) increased $(p=0,0004$ and 0,00002 , respectively). Observed changes were similar among genders.

Table 3 Characteristic of the study group and the control group. ALT - alanine transaminose, AST-aspartote transaminase. In Mann-Whitney $U$ test there are no significant differences between female and male in presented parometers. Dota ore median (interquartile ronge).

\begin{tabular}{|c|c|c|c|c|}
\hline & CONTROL & TO & T1 & $\mathrm{T} 2$ \\
\hline Total number & 33 & 45 & - & 29 \\
\hline Female & 11 & 21 & - & 11 \\
\hline Male & 22 & 24 & - & 18 \\
\hline Age, years & $47(31-65)$ & $62(56-68)$ & - & $62(51-70)$ \\
\hline Female & 52 & 66 & - & 66 \\
\hline Male & 38 & 60 & - & 61 \\
\hline Estimated time of infection, years & - & $37(33-41)$ & - & - \\
\hline Female & - & 37 & - & - \\
\hline Male & - & 36 & - & - \\
\hline Number of patients with liver cirrhosis & 7 & 30 & - & 15 \\
\hline Female & 2 & 15 & - & 6 \\
\hline Male & 5 & 15 & - & 9 \\
\hline Liver fibrosis, $\mathrm{kPa}$ & $6,0(4,3-12,0)$ & $24,8(8,5-36,3)$ & $16,2(6,8-27,4)$ & $9,0(6,2-23,3)$ \\
\hline Female & 5,5 & 24,8 & 16,8 & 11,6 \\
\hline Male & 6,8 & 25,4 & 15,9 & 8,8 \\
\hline White blood cells $\times 10^{3} \mathrm{WBC} / \mathrm{mm}^{3}$ & $6,5(5,7-7,0)$ & $5,0(4,3-6,2)$ & $6,1(5,1-7,3)$ & $6,0(5,5-7,7)$ \\
\hline Female & 6,0 & 4,9 & 6,4 & 6,0 \\
\hline Male & 6,8 & 5,3 & 6,1 & 6,4 \\
\hline Haemoglobin, g/dl & $14,3(13,1-15,2)$ & $13,7(12,3-14,8)$ & $13,7(12,9-14,9)$ & $14,0(13,4-15,2)$ \\
\hline Female & 13,1 & 13,5 & 13,6 & 13,6 \\
\hline Male & 14,8 & 14,1 & 14,4 & 14,6 \\
\hline Platelet count, $\times 10^{3}$ platelets $/ \mathrm{mm} 3$ & $225(176-259)$ & $109(75-159)$ & $130(92-189)$ & $132(101-203)$ \\
\hline Female & 244 & 105 & 113 & 124 \\
\hline Male & 214 & 111 & 137 & 153 \\
\hline ALT level, IU/mL & $29(19-39)$ & $58(42-97)$ & $21(17-31)$ & $20(15-25)$ \\
\hline Female & 36 & 69 & 23 & 21 \\
\hline Male & 29 & 57 & 19 & 20 \\
\hline AST level, IU/mL & $24(20-29)$ & $60(37-78)$ & $27(20-36)$ & $21(17-28)$ \\
\hline Female & 27 & 72 & 30 & 22 \\
\hline Male & 23 & 51 & 24 & 21 \\
\hline Total bilirubin, $\mu \mathrm{mol} / \mathrm{l}$ & $9,3(6,8-12,2)$ & $14,3(10,3-21,7)$ & $10,3(7,4-17,8)$ & $9,0(7,7-10,7)$ \\
\hline Female & 7,2 & 14,3 & 11,3 & 9,7 \\
\hline Male & 10,3 & 14,8 & 9,0 & 9,0 \\
\hline
\end{tabular}

There were significant changes in the expression of NK receptors and their MFI after the treatment. 
First of all, the percentage of NK cells among all lymphocytes was significantly lower in the group of chronically infected comparing with the control group $(p=0,004)$. Treatment caused the increase in this percentage in both $\mathrm{T} 1$ and $\mathrm{T} 2$ ( $\mathrm{p}=0,01$ and 0,03 , respectively). There were no differences between the control group and the cured population.

We assessed three activating NK receptors: NKG2D, NKp30 and KIR2DS4. Soley expression of KIR2DS4 was elevated on NK cells in patients with $\mathrm{CHC}(\mathrm{p}=0,009)$, and it remained increased after the treatment $(p=0,03$ in T1). Mean Fluorescence Intensity (MFI) of NKp30 was significantly increased in patients with $\mathrm{CHC}$ comparing with controls $(p=0,04)$, and there was a visible but statistically insignificant increase in MFI of KIR2DS4. After the treatment intensity of fluorescence of those receptors decreased in T1 $(p=0,007$ and 0,0005 , consecutively), while in T2 we observed diminished expression only in KIR2DS4 ( $p=0,006)$.

We analysed two inhibitory receptors: NKG2A and KIR2DL2/DL3 (CD158b). We did not observe any changes in their expression on NK cells, regardless of HCV status. MFI of NKG2A increased significantly among $\mathrm{CHC}$ patients comparing to controls $(\mathrm{p}=0,0006), \mathrm{CD} 158$ was elevated without statistical evidence. After the therapy, MFI of both receptors decreased ( $p=0,0004$ and 0,003 in $T 1 ; p=0,0006$ and 0,03 in T2, consecutively).

To assess two mechanisms of NK cells cytotoxicity we measured expression and MFI of TRAIL protein and granzyme $B$. In the study group, we observed increase of granzyme $B(p=0,02$ in T1) and decrease of TRAIL expression on NK cells ( $p=0,04$ in T2) after the DAA treatment.

Finally, with ELISA technique, we checked serum levels of NK-chemoattractant chemokine, CXCL10. It was significantly elevated among chronically HCV-infected patients $(p=0,001)$, and it decreased after the therapy ( $p=0,001$ in $\mathrm{T} 1$ and 0,00003 in T2).

Summary of described changes is presented in Table 3. 


\begin{tabular}{|c|c|c|c|c|}
\hline & CONTROL & TO & $\mathrm{T} 1$ & $\mathrm{~T} 2$ \\
\hline NK/sample \% & 3,2 & 2,7 & 2,8 & 2,9 \\
\hline NK/lymph \% & 15,0 & $\underline{9,1}$ & 12,7 & 11,2 \\
\hline NK GRANZ B $\%$ & 88,6 & 88,4 & 92,7 & 90,1 \\
\hline GRANZ B MFI & 7205,0 & 6872,0 & 7109,0 & 6918,0 \\
\hline NK KIR2DS4+ 6 & 1,7 & $\underline{6,8}$ & $\underline{6,9}$ & 3,1 \\
\hline NK KIR2DS4+MFI & 1416 & 1774,0 & 1546,0 & 1306,0 \\
\hline NK KIR2DL2/DL3+ $\%$ (cd158b) & 28,1 & 35,8 & 31,1 & 29,5 \\
\hline NK KIR2DL2/DL3+MFI & 5142,0 & 6293,0 & 4199,0 & 4668,0 \\
\hline NK NKG2A+ $\%$ & 42,0 & 43,7 & 40,4 & 38,8 \\
\hline NK NKG2A+MFI & 6454,0 & $\underline{9298,0}$ & 7328,5 & 5364,0 \\
\hline NK NKG2D+ $\%$ & 76,6 & 72,9 & 77,3 & 85,1 \\
\hline NK NKG2D+MFI & 3033,5 & 3163,0 & 2958,0 & 2669,0 \\
\hline NK TRAIL + $\%$ & 0,4 & 0,7 & 0,3 & 0,2 \\
\hline NK TRAIL+MFI & 1172,0 & 1093,0 & 999,0 & 980,0 \\
\hline NK NKp3O+ \% & 63,9 & 66,6 & 65,3 & 53,9 \\
\hline NK NKp30+ MFI & 2958,0 & $\underline{3198,5}$ & 2635,5 & $\underline{2107,5}$ \\
\hline serum $\mathrm{CXCL} 10 \mathrm{pg} / \mathrm{ml}$ & 74,8 & 140,3 & 91,1 & 64,9 \\
\hline
\end{tabular}

Table 3 Median values of measured NK cells' porameters in the healthy control group (control), in chronically HCV-infected individuals (TO) and in potients treoted with DAA in two consecutive time points ( $T 1$ and $T 2$ ). Underlined numbers differ significantly from those in the control group in Monn-Whitney $U$ test. Highlighted windows present volues that changed significantly after the successful treatment in Wilcoxon signed-rank test - blue colour indicates decrease and red means increase.

Afterwards, we compared results described above among genders. Comparing chronically infected patients with the control group, we observed, that MFI of KIR2DL2/DL3 was elevated only in female $(p=0,02)$ and MFI of NKp30 was higher in male $(p=0,016)$. There were a few significant differences in NK cells' reaction to HCV eradication between men and women. All the changes are presented in the figures below.

\section{Discussion}

After the successful DAA therapy, we observed a decrease in liver fibrosis as well as improvement in biochemical and haematological parameters comparable to the changes presented in the literature ${ }^{33}$. Interferon-free HCV therapies are doubtlessly a great achievement of modern medicine.

In the group of patients witch $\mathrm{CHC}$, we observed changes in NK cells that partially restored after the virus eradication. Generally, the modifications were consistent with those described in other studies, summed up in table 1. We showed a pattern of changes of activating receptor KIR2DS4 that, with our best knowledge, have not been described in the context of $\mathrm{CHC}$ and its' treatment before. This finding enlightens our understanding of the behaviour of NK cells in the course of HCV infection.

Our data showed few differences between genders in NK cells' reaction to the successful treatment of $\mathrm{CHC}$. 
NKp30 is a member of Natural Cytotoxicity Receptors family, that is expressed on all mature resting and activated NK cells. This activating receptor plays a central role in resistance to infectious diseases ${ }^{34}$. High baseline expression of this receptor favours vivid NK cells reaction to infections like e.g. malaria ${ }^{35}$. It is most likely hormone-dependent since it's expression varies in different phases of menstrual cycle ${ }^{36}$. In various studies, it was either increased or decreased in chronically HCV-infected patients, remaining its behaviour in given disease elusive. There is a theory that in chronically infected patients it persists increased, nonetheless inactive ${ }^{37}$. Researches showed NKp30 decreases among patients who cleared HCV spontaneously in the acute phase, below the values of healthy population ${ }^{16}$. In our study, MFI of NKp30 was increased only in chronically infected male, not in the female. After the treatment, it decreased significantly in women, leaving them with final values lower than in healthy controls. This result may show that female response to infectious diseases may be diminished even after the successful HCV treatment.

Killer cell immunoglobulin-like receptors (KIRs) repertoire of NK cells depends on both KIR and HLA gene polymorphisms ${ }^{38}$. Depending on KIR genotype, male and female have a different probability of becoming chronically HCV-infected ${ }^{39}$. Haplotype A of KIRs includes KIR2DL2/DL3 (CD158b), an inhibitory receptor that senses HLA-1 expressing cells and prevents NK cells from auto aggression. Its excessive activation causes susceptibility to malignancies like melanoma and its metastasis ${ }^{38}$. In children with $\mathrm{CHC}$ it is associated with more advanced liver fibrosis ${ }^{40}$. There is a link between KIR2DL2 genes and HCV-related lymphoproliferative disorders ${ }^{41}$. Combination of KIR2DL2 presence with HLA-C1 is a significant risk factor of HCC development in HCV-infected patients ${ }^{42}{ }^{43}$. Some papers show the receptor's increase in chronic HCV infection, while others prove it remains unchanged. Our data indicate that MFI of CD158b on NK cells increases in HCV-infected female comparing to healthy controls, and normalises after the successful treatment. In the male expression of that receptor remained unchanged in all examined groups, regardless of infection status. In our opinion, its expression in $\mathrm{CHC}$ requires further studies.

Activation-induced NK cell death is accompanied by the leakage of granzyme B from intracellular granules into the cytoplasm. This serine protease contributes to target cell death, playing an important role in the development of autoimmunity, e.g., rheumatoid arthritis ${ }^{44}$. T-cells of patients with HCC present diminish the production of granzyme $\mathrm{B}^{45}$. Due to its antigen-specific properties, this protease is considered novel therapy for solid tumors ${ }^{46}$. In our study percentage of NK cells expressing granzyme $B$ did not differ between chronically HCV-infected patients and controls but increased in treated men, not women. This finding may suggest male are better protected by NK cells against HCC and autoimmunity after the completed therapy. Since statistically among them recurrence and occurrence rate of HCC is higher ${ }^{28}$, immunological mechanisms of this phenomenon require further investigations.

C-X-C chemokine 10 (CXCL10) is a chemokine produced by hepatocytes and liver-infiltrating lymphocytes during HCV infection, inducing the migration of T-cells and NK-cells to the liver ${ }^{47}$. High levels of CXCL10 are connected to poor treatment outcomes in patients with chronic hepatitis $\mathrm{C}$ and decompensated 
cirrhosis treated with $D_{A A}{ }^{48}$. Furthermore, elevated serum concentrations of this chemoattractant are associated with HCV-related mixed cryoglobulinaemia, especially in patients with clinically active vasculitis. ${ }^{49}$ Female gender is one of the main factors related to cryoglobulin production ${ }^{50}$. Interferon-free therapies seem to decrease vasculitis activity in most HCV-patients ${ }^{51,52}$. In our study, CXCL10 levels declined in women after the therapy, which confirms the significant role of CXCL10 in the pathogenesis of HCV-related mixed-cryoglobulinemia. CXCL10 is speculated to be a candidate for a novel therapeutic target in this condition ${ }^{49}$, and our study supports this concept.

In conclusion, post-menopausal female and male differ in the reaction of their NK cells to interferon-free therapy of $\mathrm{CHC}$. Our research may lead to more studies on different nature of female and male immune systems in the context of HCV infection and treatment.

\section{Declarations}

\section{Ethics Statement}

This study was carried out following the Declaration of Helsinki of the World Medical Association and was approved by the Ethical Committee of Poznan University of Medical Sciences. All the enrolled study participants met the criteria and completed the study. They were fully informed about the study, and all of them expressed written informed consent before their examination.

\section{Acknowledgements}

This work was supported by the National Science Centre, Poland (NCN) grant no. 2016/23/B/NZ6/01497.

\section{Conflict of interest}

The authors declare that they have no conflict of interest.

\section{References}

1. Prager, I. \& Watzl, C. Mechanisms of natural killer cell-mediated cellular cytotoxicity. Journal of Leukocyte Biology 105, 1319-1329 (2019).

2. Abel, A. M., Yang, C., Thakar, M. S. \& Malarkannan, S. Natural killer cells: Development, maturation, and clinical utilisation. Frontiers in Immunology 9, (2018).

3. Yoon, J. C., Yang, C. M., Song, Y. \& Lee, J. M. Natural killer cells in hepatitis C: Current progress. World journal of gastroenterology 22, 1449-1460 (2016).

4. Castello, G., Scala, S., Palmieri, G., Curley, S. A. \& Izzo, F. HCV-related hepatocellular carcinoma: From chronic inflammation to cancer. Clinical Immunology 134, 237-250 (2010).

5. Jinushi, M. et al. Negative Regulation of NK Cell Activities by Inhibitory Receptor CD94/NKG2A Leads to Altered NK Cell-Induced Modulation of Dendritic Cell Functions in Chronic Hepatitis C Virus 
Infection. J. Immunol. 173, 6072-6081 (2004).

6. Podhorzer, A. et al. The clinical features of patients with chronic hepatitis $C$ virus infections are associated with killer cell immunoglobulin-like receptor genes and their expression on the surface of natural killer cells. Front. Immunol. 8, (2018).

7. Oliviero, B. et al. Natural Killer Cell Functional Dichotomy in Chronic Hepatitis B and Chronic Hepatitis C Virus Infections. Gastroenterology 137, (2009).

8. Serti, E. et al. Successful Interferon-Free Therapy of Chronic Hepatitis C Virus Infection Normalises Natural Killer Cell Function. Gastroenterology 149, 190-200.e2 (2015).

9. Szereday, L. et al. Immunological changes in different patient populations with chronic hepatitis $\mathrm{C}$ virus infection. World J. Gastroenterol. 22, 4848-4859 (2016).

10. Séne, D. et al. Hepatitis C virus (HCV) evades NKG2D-dependent NK cell responses through NS5Amediated imbalance of inflammatory cytokines. PLoS Pathog. 6, (2010).

11. Dessouki, O. et al. Chronic hepatitis $C$ viral infection reduces NK cell frequency and suppresses cytokine secretion: Reversion by anti-viral treatment. Biochem. Biophys. Res. Commun. 393, 331337 (2010).

12. Spaan, M. et al. Immunological Analysis During Interferon-Free Therapy for Chronic Hepatitis C Virus Infection Reveals Modulation of the Natural Killer Cell Compartment. J. Infect. Dis. 213, 216-23 (2016).

13. De Maria, A. et al. Increased natural cytotoxicity receptor expression and relevant IL-10 production in NK cells from chronically infected viremic HCV patients. Eur. J. Immunol. 37, 445-455 (2007).

14. Perpiñán, E. et al. Cirrhosis Hampers Early and Rapid Normalisation of Natural Killer Cell Phenotype and Function in Hepatitis C Patients Undergoing Interferon-Free Therapy. Front. Immunol. 11, (2020).

15. Nattermann, J. et al. Surface expression and cytolytic function of natural killer cell receptors is altered in chronic hepatitis C. Gut 55, 869-877 (2006).

16. Ji, H. F. et al. High frequencies of CD158b+ NK cells are associated with persistent hepatitis $C$ virus infections. Ann. Hepatol. 12, 371-379 (2013).

17. Ahlenstiel, G. et al. Natural Killer Cells Are Polarised Toward Cytotoxicity in Chronic Hepatitis C in an Interferon-Alfa-Dependent Manner. Gastroenterology 138, (2010).

18. Strunz, B., Hengst, J., Wedemeyer, H. \& Bjorkstrom, N. K. Irreversible impact of chronic hepatitis C virus infection on human natural killer cell diversity. Cell Stress 2, 216-218 (2018).

19. Abdel-Samie, E. R. et al. Effect of Combined HCV Therapy on Natural Killer Cell Activity. Egypt J Immunol 26, 151-161(2019).

20. Jaillon, S., Berthenet, K. \& Garlanda, C. Sexual Dimorphism in Innate Immunity. Clinical Reviews in Allergy and Immunology 56, 308-321 (2019).

21. Phan, M. T. et al. Natural killer cell subsets and receptor expression in peripheral blood mononuclear cells of a healthy Korean population: Reference range, influence of age and sex, and correlation between NK cell receptors and cytotoxicity. Hum. Immunol. 78, 103-112 (2017). 
22. Giefing-Krö, C., Berger, P. \& Grubeck-Loebenstein, B. How sex and age affect immune responses, susceptibility to infections, and response to vaccination. doi:10.1111/acel.12326

23. Al-Attar, A., Presnell, S. R., Peterson, C. A., Thomas, D. T. \& Lutz, C. T. The effect of sex on immune cells in healthy aging: Elderly women have more robust natural killer lymphocytes than do elderly men. Mech. Ageing Dev. 156, 25-33 (2016).

24. Jilkova, Z. M. et al. Sex Differences in Spontaneous Degranulation Activity of Intrahepatic Natural Killer Cells during Chronic Hepatitis B: Association with Estradiol Levels. (2017). doi:10.1155/2017/3214917

25. Ruggieri, A., Gagliardi, M. C. \& Anticoli, S. Sex-dependent outcome of hepatitis B and C Viruses infections: Synergy of sex hormones and immune responses? Frontiers in Immunology 9, (2018).

26. Ruggieri, A., Barbati, C. \& Malorni, W. Cellular and molecular mechanisms involved in hepatocellular carcinoma gender disparity. International Journal of Cancer 127, 499-504 (2010).

27. Mozer-Lisewska, I. et al. Genetic (KIR, HLA-C) and Some Clinical Parameters Influencing the Level of Liver Enzymes and Early Virologic Response in Patients with Chronic Hepatitis C. Arch. Immunol. Ther. Exp. (Warsz). 64, 65-73 (2016).

28. Rinaldi, L. et al. Incidence and risk factors of early HCC occurrence in HCV patients treated with direct acting antivirals: A prospective multicentre study. J. Transl. Med. 17, (2019).

29. Halota, W. et al. HEPATOLOGIA 2018 Rekomendacje Polskiej Grupy Ekspertów HCV dotyczące leczenia wirusowych zapaleń wątroby typu C w roku 2018 Recommendations by Polish Group of Experts for HCV for the treatment of viral hepatitis C in 2018. HEPATOLOGIA 18, 1-9 (2018).

30. Jung, K. S. \& Kim, S. U. Clinical applications of transient elastography. Clin. Mol. Hepatol. 18, 163 (2012).

31. Kierepa, A. et al. Impact of chronic HCV treatment on quality of life of patients with metabolic disorders in context of immunological disturbances. Sci. Rep. 10, 1-10 (2020).

32. Skinner, J. Statistics for Immunologists.

33. Hsu, W. F. et al. Rapid decline of noninvasive fibrosis index values in patients with hepatitis $\mathrm{C}$ receiving treatment with direct-acting antiviral agents. BMC Gastroenterol. 19, 63 (2019).

34. Barrow, A. D., Martin, C. J. \& Colonna, M. The natural cytotoxicity receptors in health and disease. Frontiers in Immunology 10, 909 (2019).

35. Walk, J. \& Sauerwein, R. W. Activatory Receptor NKp30 Predicts NK Cell Activation During Controlled Human Malaria Infection. Front. Immunol. 10, 2864 (2019).

36. Ponnampalam, A. P., Gargett, C. E. \& Rogers, P. A. W. Identification and hormonal regulation of a novel form of NKp30 in human endometrial epithelium. Eur. J. Immunol. 38, 216-226 (2008).

37. Marras, F., Bozzano, F., Ascierto, M. L. \& de Maria, A. Baseline and dynamic expression of activating NK cell receptors in the control of chronic viral infections: The paradigm of HIV-1 and HCV. Frontiers in Immunology 5, (2014). 
38. Konjevic, G. et al. Investigation of NK cell function and their modulation in different malignancies. Immunol. Res. 52, 139-156 (2012).

39. Kuśnierczyk, P. et al. Contribution of genes for killer cell immunoglobulin-like receptors (KIR) to the susceptibility to chronic hepatitis C virus infection and to viremia. Hum. Immunol. 76, 102-108 (2015).

40. Mania, A. et al. Expression of natural killer cell inhibitory receptors is associated with significant liver injury in chronic hepatitis C in children. Ann. Hepatol. 16, 521-529 (2017).

41. De Re, V. et al. Genetic diversity of the KIR/HLA system and susceptibility to hepatitis C virus-related diseases. PLoS One 10, (2015).

42. Saito, H. et al. KIR2DL2 combined with HLA-C1 confers risk of hepatitis C virusrelated hepatocellular carcinoma in younger patients. Oncotarget 9, 19650-19661 (2018).

43. Cariani, E. et al. HLA and killer immunoglobulin-like receptor genes as outcome predictors of hepatitis C virus-related hepatocellular carcinoma. Clin. Cancer Res. 19, 5465-5473 (2013).

44. Ida, H., Utz, P. J., Anderson, P. \& Eguchi, K. Granzyme B and natural killer (NK) cell death. Modern Rheumatology 15, 315-322 (2005).

45. Kalathil, S., Lugade, A. A., Miller, A., Iyer, R. \& Thanavala, Y. Higher frequencies of GARP+CTLA$4+F o x p 3+t$ regulatory cells and myeloid-derived suppressor cells in hepatocellular carcinoma patients are associated with impaired t-cell functionality. Cancer Res. 73, 2435-2444 (2013).

46. Qian, X. et al. A novel Granzyme B nanoparticle delivery system simulates immune cell functions for suppression of solid tumors. Theranostics 9 , 7616-7627 (2019).

47. Riva, A. et al. Truncated CXCL10 is associated with failure to achieve spontaneous clearance of acute hepatitis C infection. Hepatology 60, 487-496 (2014).

48. Childs, K. et al. Open Forum Infectious Diseases Open Forum Infectious Diseases ® Immunological Predictors of Nonresponse to Directly Acting Antiviral Therapy in Patients With Chronic Hepatitis C and Decompensated Cirrhosis. (2017). doi:10.1093/ofid/ofx067

49. Mazzi, V. et al. Role of CXCL10 in cryoglobulinaemia. Clinical and Experimental Rheumatology 33, 433-436 (2015).

50. Cacoub, P. \& Saadoun, D. Hepatitis C virus infection induced vasculitis. Clinical Reviews in Allergy and Immunology 35, 30-39 (2008).

51. Mazzaro, C. et al. Long-term effects of the new direct antiviral agents (DAAs) therapy for HCV-related mixed cryoglobulinaemia without renal involvement: a multicentre open-label study. Clin. Exp. Rheumatol. 36 Suppl 111, 107-114

52. Sise, M. E. et al. Treatment of hepatitis $C$ virus-associated mixed cryoglobulinemia with direct-acting antiviral agents. Hepatology 63, 408-417 (2016).

\section{Figures}



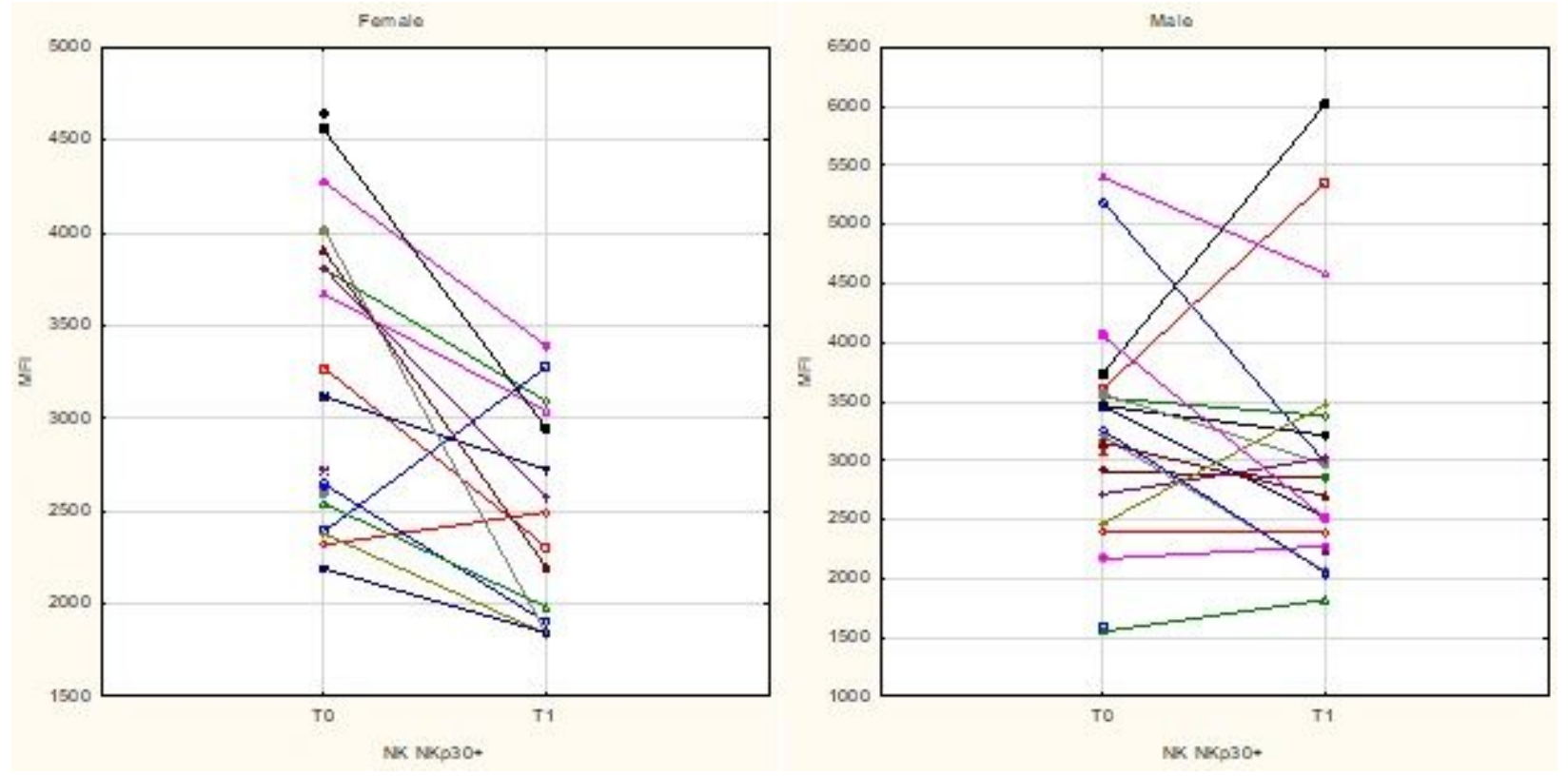

\section{Figure 1}

MFI of NKp30 on NK cells before (T0) and after (T1) the treatment in female and male. In Wilcoxon signed-rank test, mean MFI in female decreased significantly $(p=0,01)$ while in male did not change.
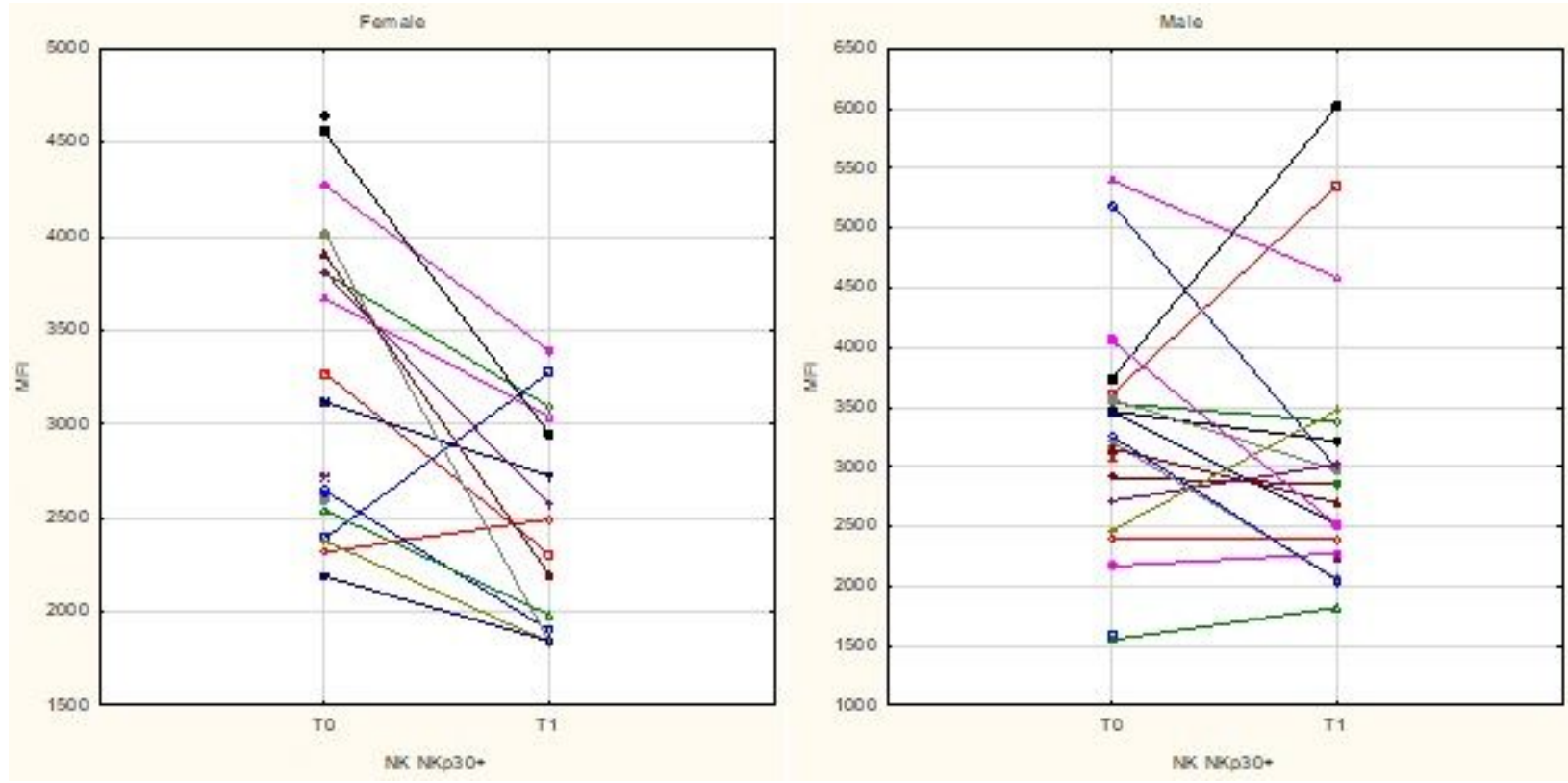

Figure 1

MFI of NKp30 on NK cells before (T0) and after (T1) the treatment in female and male. In Wilcoxon signed-rank test, mean MFI in female decreased significantly $(p=0,01)$ while in male did not change. 

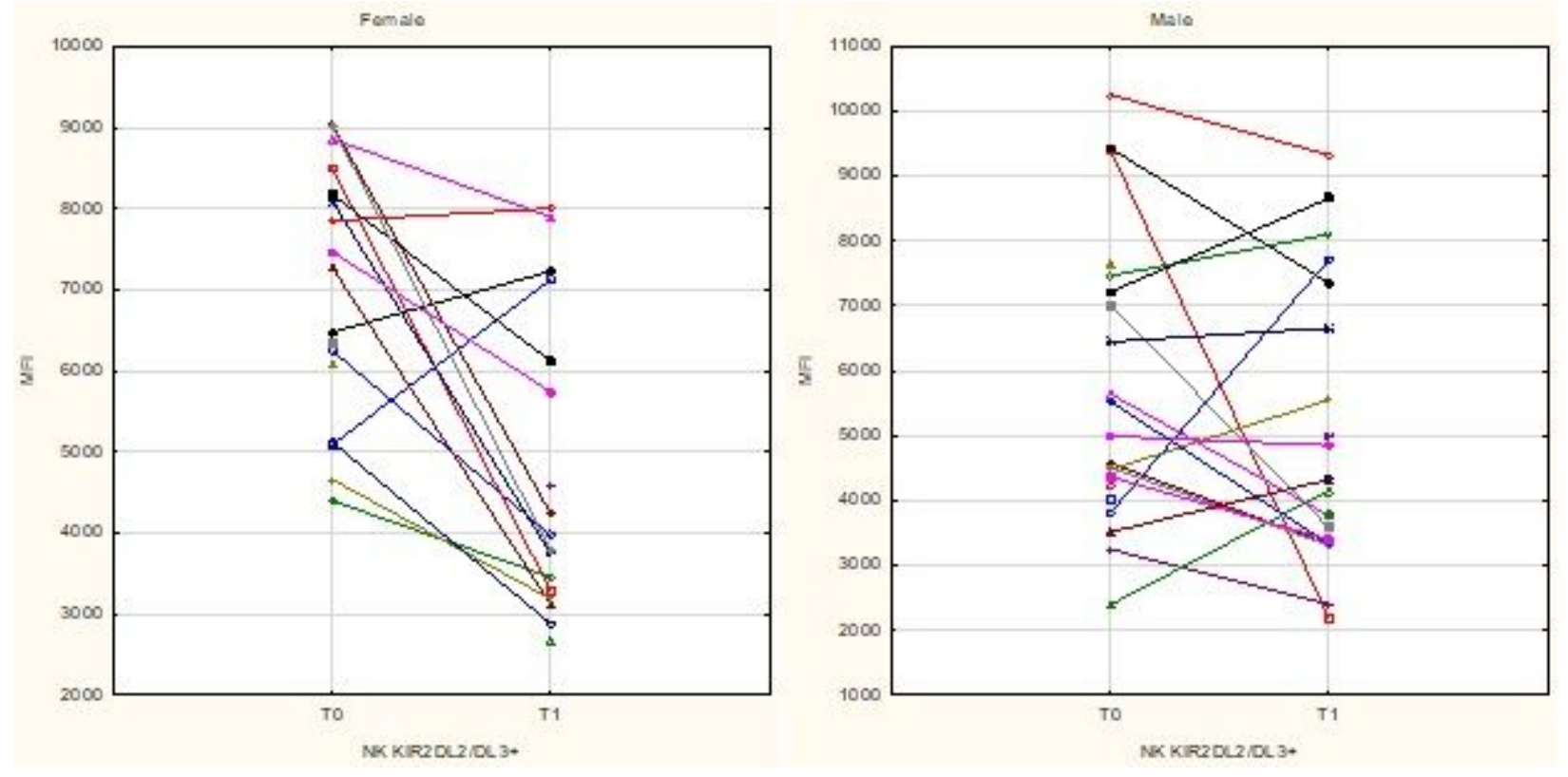

\section{Figure 2}

MFI of KIR2DL2/DL3 on NK cells before (T0) and after (T1) the treatment in female and male. In Wilcoxon signed-rank test, mean MFI in female decreased significantly $(p=0,04)$ while in male did not change.
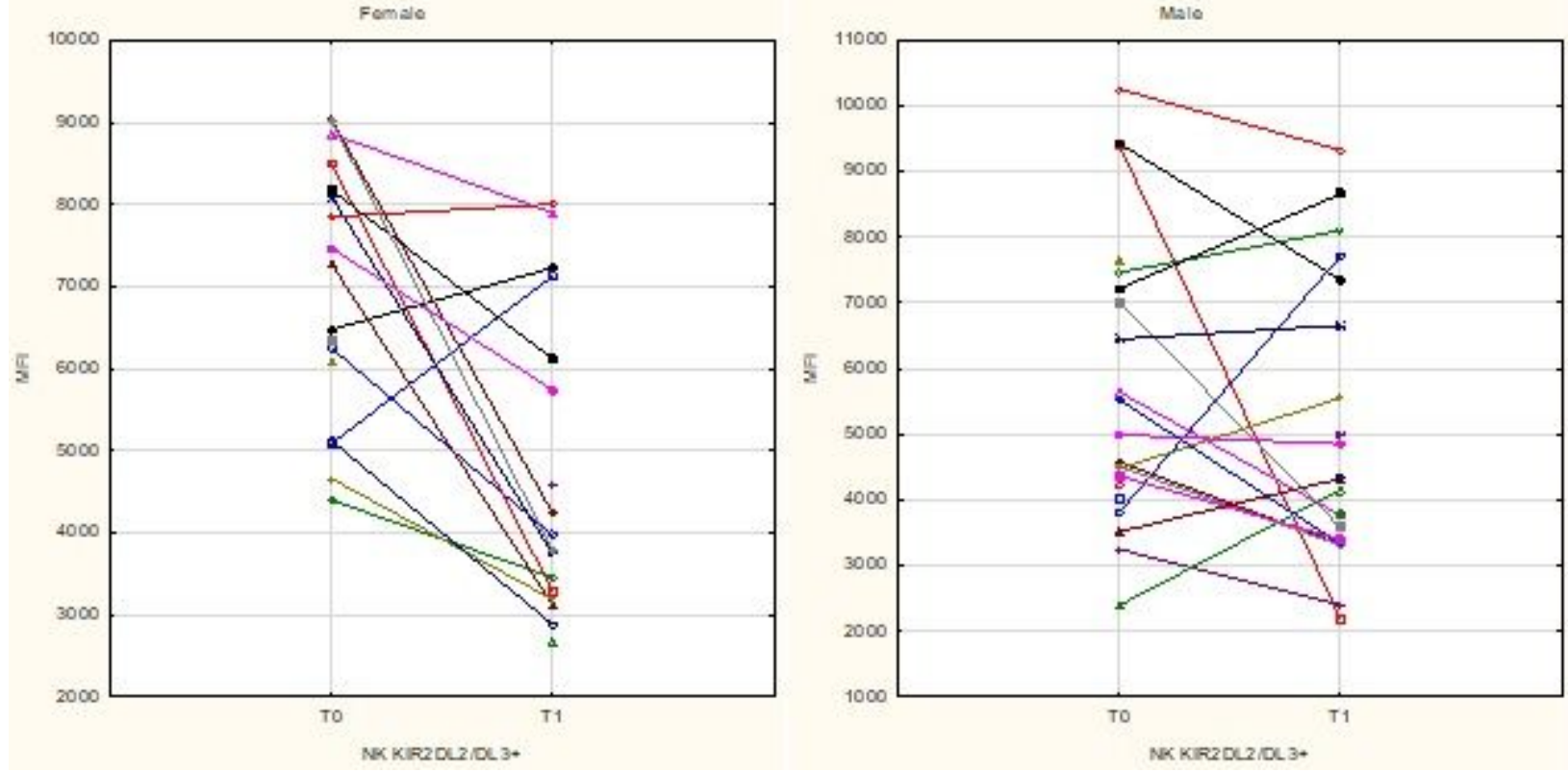

Figure 2

MFI of KIR2DL2/DL3 on NK cells before (T0) and after (T1) the treatment in female and male. In Wilcoxon signed-rank test, mean MFI in female decreased significantly $(p=0,04)$ while in male did not change. 

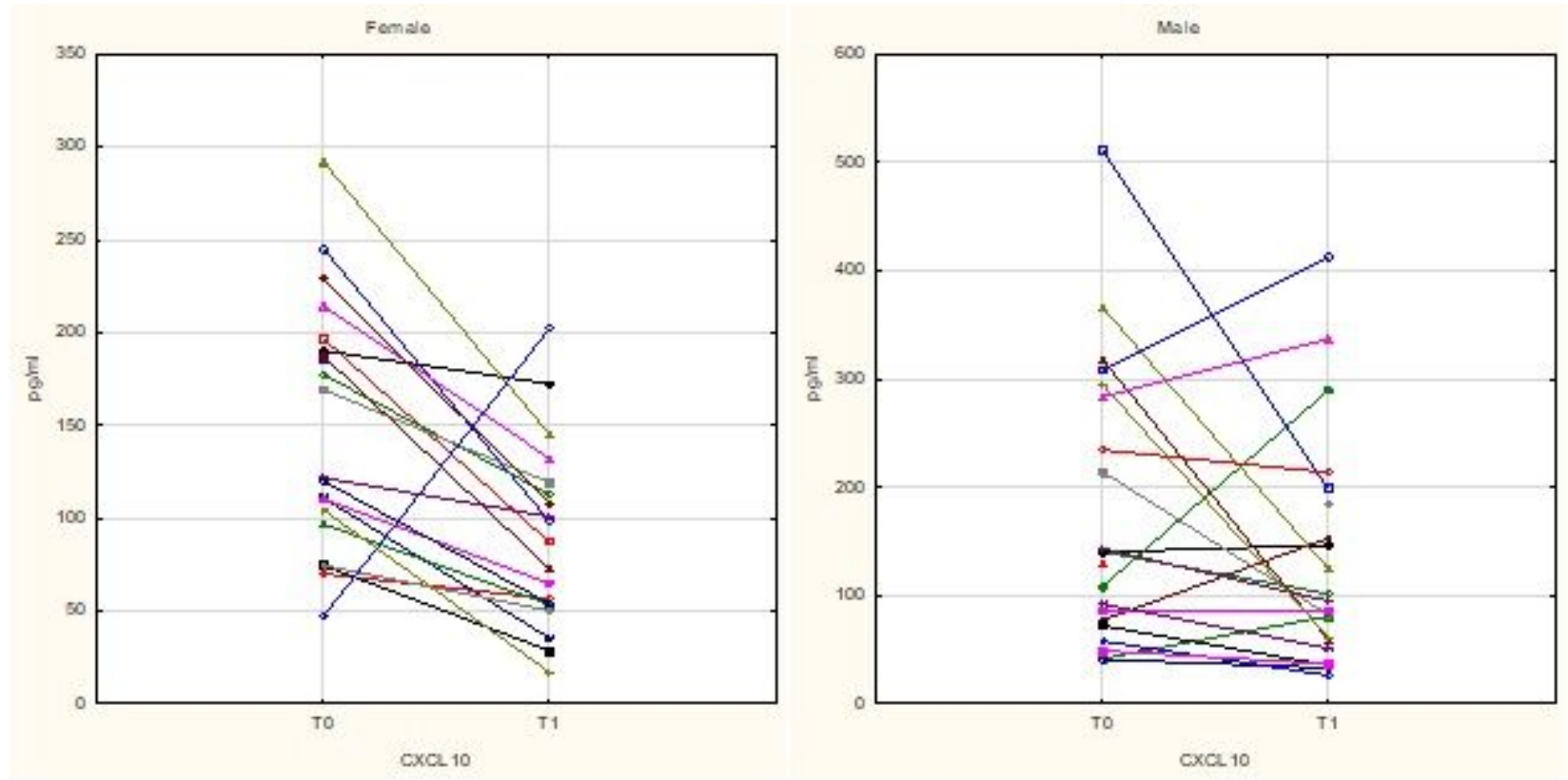

Figure 3

Serum levels of CXCL10 before (T0) and after (T1) the treatment in female and male. In Wilcoxon signedrank test, mean concentration in female decreased significantly $(p=0,002)$ while in male did not change.
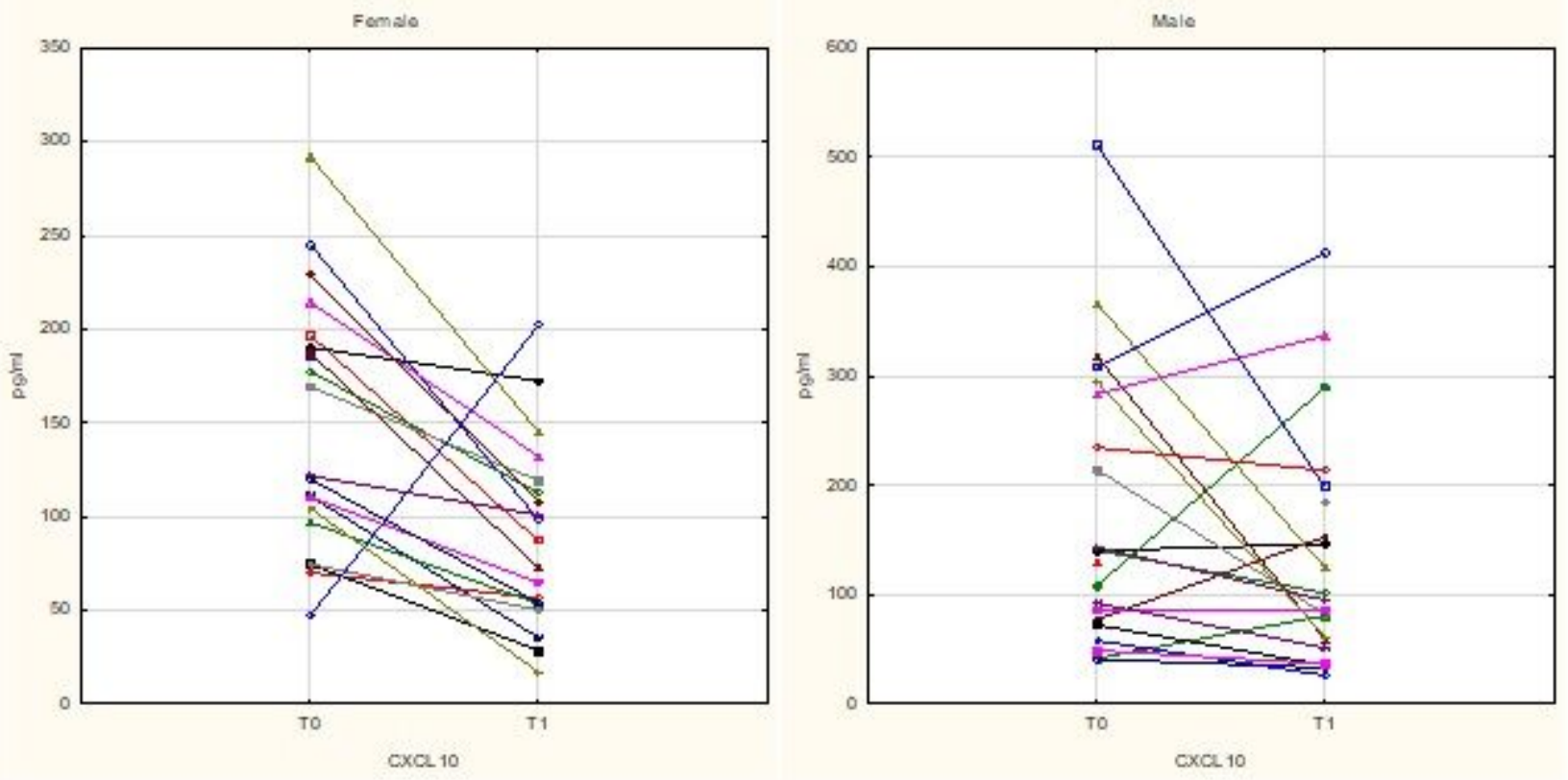

Figure 3

Serum levels of CXCL10 before (T0) and after (T1) the treatment in female and male. In Wilcoxon signedrank test, mean concentration in female decreased significantly $(p=0,002)$ while in male did not change. 

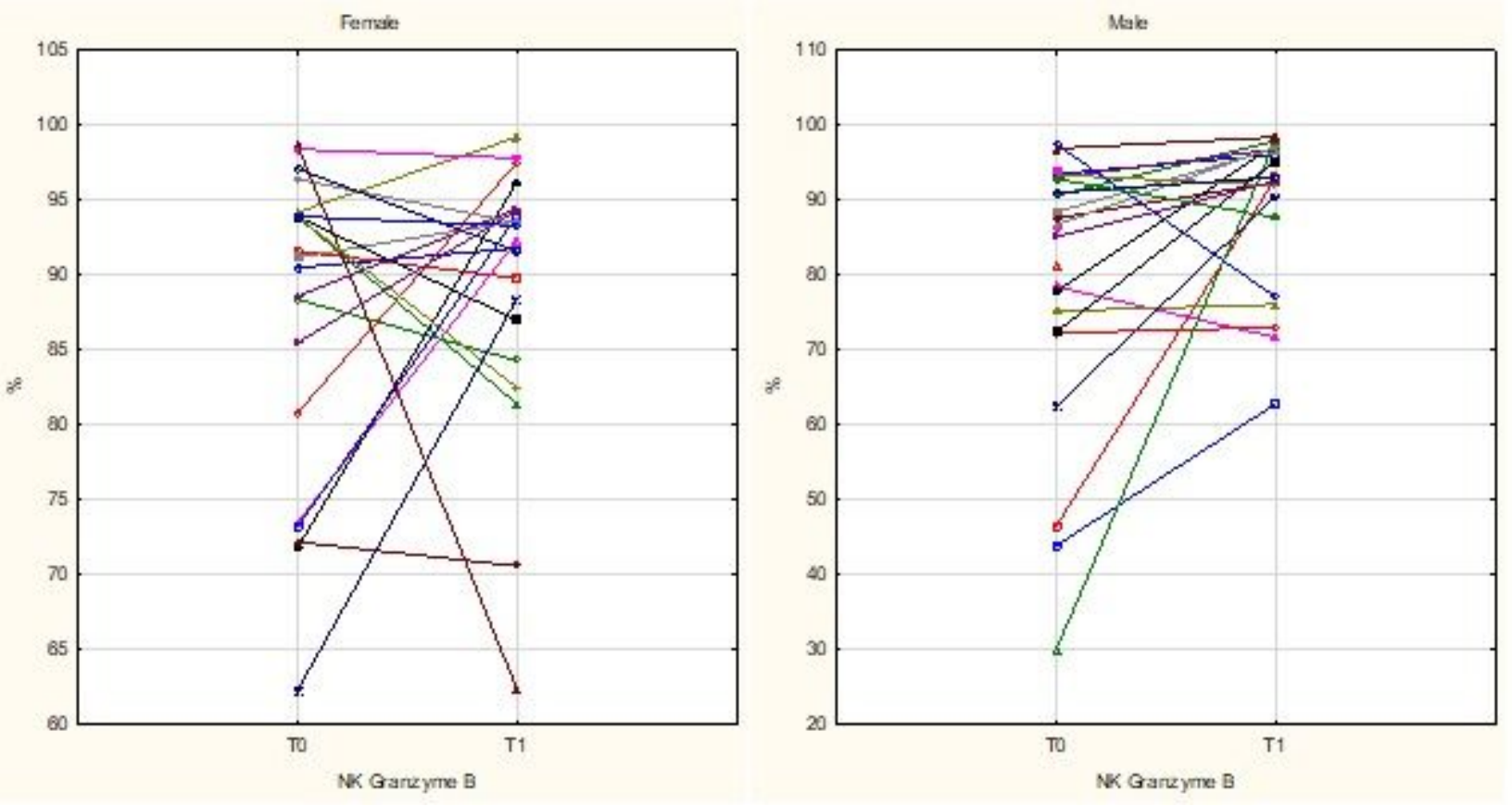

\section{Figure 4}

Percentage of NK cells expressing granzyme B in female and male before DAA treatment (T0) and after (T1). In Wilcoxon signed-rank test mean percentage in male increased significantly $(p=0,008)$ while in female did not change.
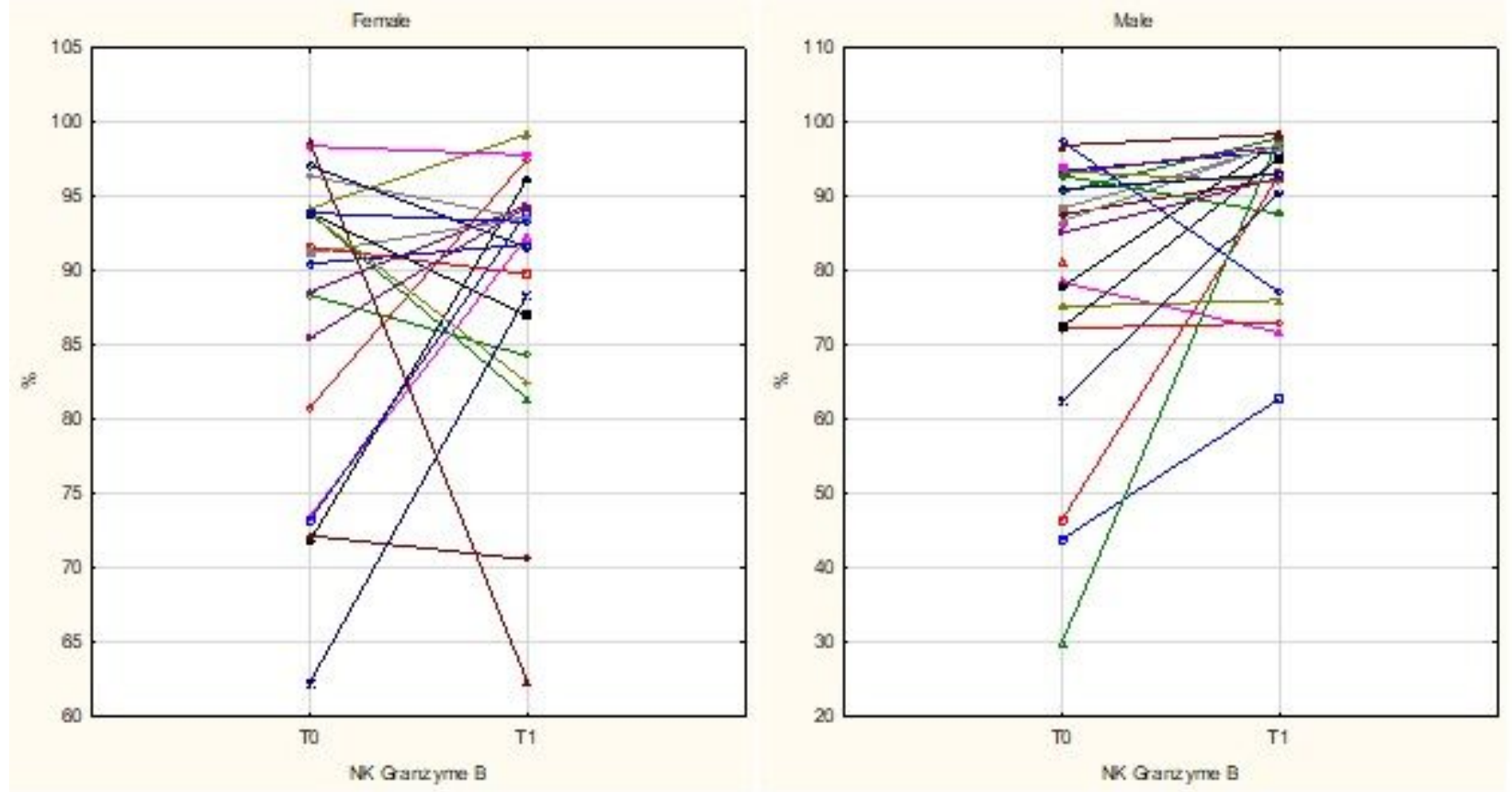

Figure 4

Percentage of NK cells expressing granzyme B in female and male before DAA treatment (TO) and after (T1). In Wilcoxon signed-rank test mean percentage in male increased significantly $(p=0,008)$ while in female did not change. 